\title{
Mass, Impulse, Force, Energy transfer via Quantum Entanglement in linear pitch
}

\author{
Boris Lagutin
}

Funding: The author(s) received no specific funding for this work.

Potential competing interests: The author(s) declared that no potential competing interests exist.

\section{Abstract}

In this paper (preprint), the author analyzes how physically (not mathematically) a mass of one of two impacting bodies (metal block and metal ball) may influence a speed (magnitude of velocity) after an impact of the ball. The impact happens when the block gradually accelerates the ball. The impact ends when the block is completely stopped but the ball continues to fly because of its inertia. In according to the classical mechanics formulas, the ball speed after the impact increases if a mass of the block increases. That is, it is enough to only increase a mass of the block in order to increase a final speed of the ball, keeping the same acceleration given by the block to the ball. This is also supported by the kinetic energy transfer from the block to the ball because the kinetic energy depends on a mass. The paper also describes an experiment to check how a final speed of the ball changes depending on a mass of the block. Based on the current physics, interactions between particles of the block and of the ball in an impact area cannot change depending on a mass of the block. Therefore, there is a contradictory situation that the formulas give the ball final speed increase that depends on an additional mass of the block, but the classical mechanics does not explain how the interactions between the particles can cause the speed increase.

If the speed increase after the impact is confirmed experimentally, the author assumes that the interactions between the particles may be based on the quantum entanglement relations (without fields), or the fields of the particles are quantumly entangled. In other words, the kinetic energy is transferred via some quantum entanglement interactions. The entanglement of the particles or their fields gives a chance to explain how the increase of the mass can give the speed increase. However, a series of appropriate experiments is needed to figure it out properly

Introduction

The force in the classical mechanics is defined by a mass and an acceleration of a mass. For example, a magnitude of normal force $N$ is equal to a magnitude of force $F$ exerted on a floor by a metal block, see Figure 1 , if the block does not move down [1]. 


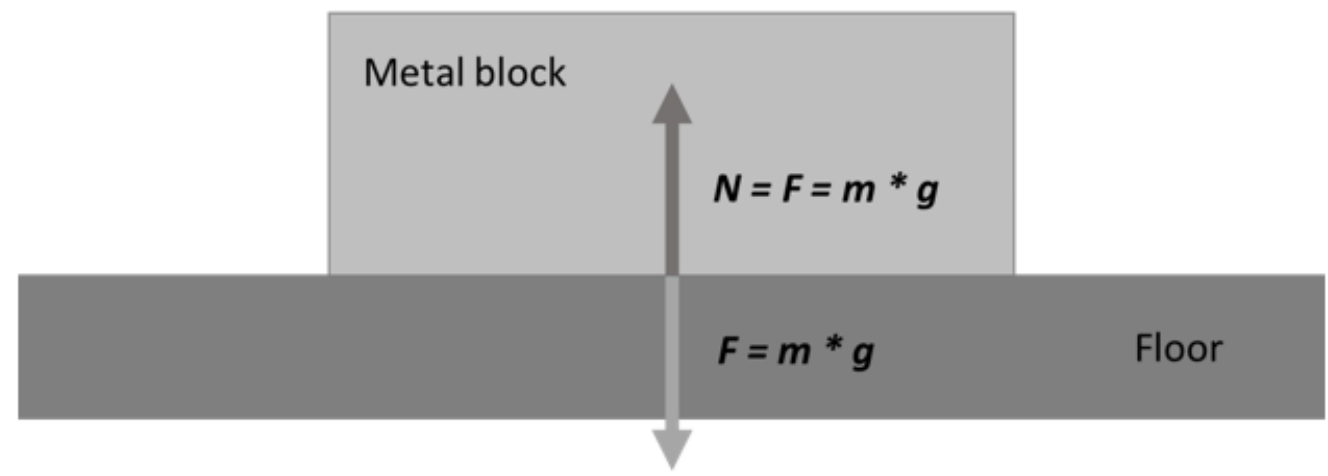

The equation for this situation is the following:

$$
|N|=|F|=|m g|,(1)
$$

where $m$ is a mass of the metal block, $g$ is a gravitational acceleration. This physical situation illustrates what the force is in the classical mechanics - a mass is multiplied by an acceleration. The classical mechanics formula of the force via impulse (difference of initial and final momenta) is [2]:

$$
F=m a=\left(m V_{\text {final }}-m V_{\text {initial }}\right) / t_{\text {impact }},(2)
$$

where $m$ is a mass of a body, $a$ is an acceleration of a body, $V$ defines a final and an initial velocities (velocities after and before impact appropriately) of a body, $t$ is time of impact.

Next watch a video about shot put:

The pitcher throws the heavy ball by his hand. It is time to remember Newton's third law applying it for the pitcher's hand throwing the ball. The law says that a force (its magnitude), which the pitcher's hand exerts on the ball, is equal to a force (its magnitude), that the ball exerts on the pitcher's hand. Here is the formula for this law in absolute values [3]:

$$
m_{\text {hand }}\left|a_{\text {hand }}\right|=m_{\text {ball }}\left|a_{\text {ball }}\right|,(3)
$$

where $m$ symbolizes masses of the pitcher's hand (ignore the pitcher's whole body for simplicity) and of the ball appropriately, a symbolizes accelerations of the pitcher's hand and of the ball. Therefore,

$$
m_{\text {hand }}\left|a_{\text {hand }}\right|=m_{\text {ball }}\left|a_{\text {ball }}\right|=|F|=\left(m_{\text {ball }}\left|V_{\text {final }}\right|-m_{\text {ball }}\left|V_{\text {initial }}\right|\right) / t_{\text {impact }}=\left(m_{\text {ball }}\left|V_{\text {final }}\right|\right) / t_{\text {impact }},(4)
$$

where $V$ symbolizes a final and initial velocities of the ball noticing that its initial velocity is zero.

Let's try to answer the following question: "Whether or not a final speed of the ball depends on a mass of the pitcher's hand if an acceleration given by the pitcher to the ball does not change?" Assume that the ball is thrown by different pitchers having distinct body masses.

Based on the formula (4), the heavier hand the pitcher has the higher acceleration the ball gets if an acceleration of the pitcher's hand and a mass of the ball do not change. Of course, the impact process between the ball and the hand is 
complicated and includes the pitcher's whole body. However, a mass of the pitcher's hand matters anyway. So, leave the pitcher's body aside for simplicity. It should be said that the author analyzes the impact processes between two bodies when one body accelerates another body, not when they are both at rest. By the way, a common example of the real application of Newton's third law concerns two students having different masses and seating on chairs on wheels [3]. One student pushes another student. As a result, a lighter student gets a higher speed after the push.

Now replace the pitcher's hand with a metal block assembled on a conveyor belt. To check how a final speed of a ball changes depending on a mass of the block, the author offers the following experiment. There are a stainless steel block 20 kilograms, a stainless steel ball 1 kilogram. The block is tightly assembled to the conveyor belt which is moved by a programmable electromotor. The block has an indent suitable to precisely accommodate the ball for preventing the ball from falling down because of the gravitational force, see Figure 2.

FIGURE 2

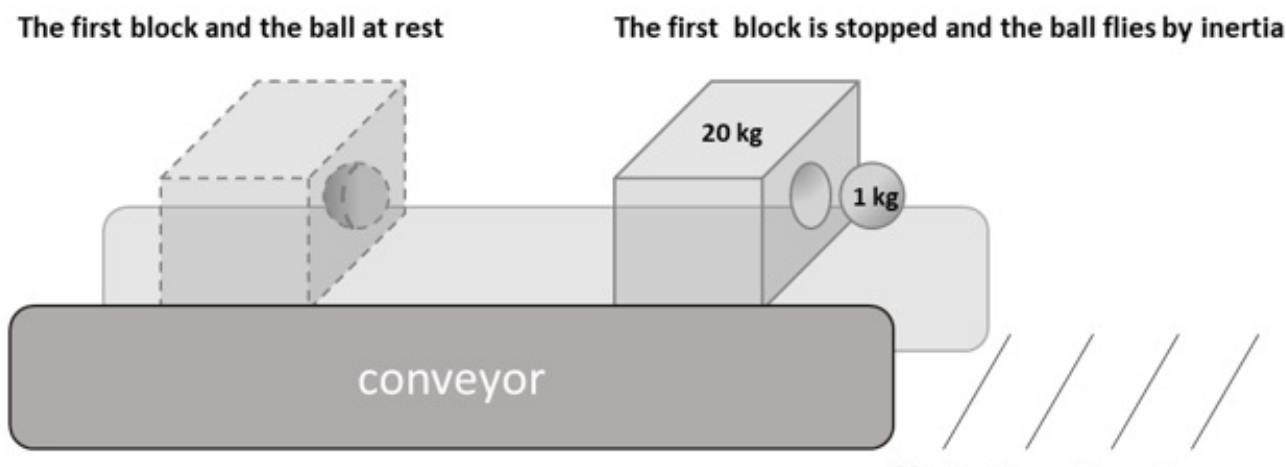

$20 \mathrm{~cm} 40 \mathrm{~cm} 60 \mathrm{~cm} 80 \mathrm{~cm}$

First, the block with the ball located in the indent are linearly accelerated up to some final speed $V$. Notice that an acceleration may be small; therefore, the block with the ball are accelerated up to $V$ for a long time so that no shock waves can happen. When a speed $V$ is reached, the block is stopped completely. As a result, the ball flies away from the block by inertia. A distance, which the ball flies over, is measured. Second, the mass of the block is increased up to 100 kilograms so that its width stays the same, or a heavier block with the same indent is used. If the heavier block is used, a height from the indent to a surface on which the ball falls down must be the same as that in the first block (block without the additional mass). The enlarged block or the heavier block with the ball in the indent are accelerated up to some final speed $V$, see Figure 3. 


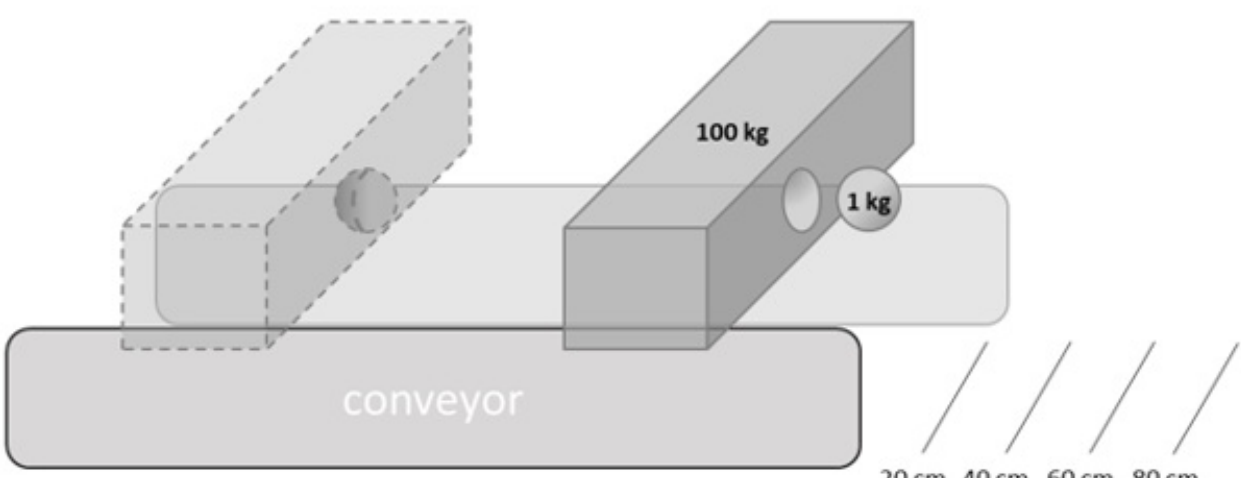

$20 \mathrm{~cm} 40 \mathrm{~cm} 60 \mathrm{~cm} 80 \mathrm{~cm}$

An acceleration and a final speed $V$ must be the same as those for the first block (20 kilograms). When the enlarged block gains a necessary final speed $V$, the enlarged block is stopped completely; hence, the ball flies away from the enlarged block by inertia. A distance, which the ball flies over, is measured.

Two video cameras are used to record the experiment. The first camera records the motions of the conveyor, the blocks, the ball during the experiment from a distance of some meters. The second camera records what happens in the impact area so that the motion of the ball can be recorded very zoomed-in millisecond by millisecond.

Conclusion

If a distance, which the ball flies over when it is accelerated by the first block, is less than a distance, which the ball flies over when it is accelerated by the enlarged block, then it is evident that the ball gets a higher final speed when the ball is accelerated by the enlarged block. The only factor, which can influence the speed increase of the ball, is the increase of the first block mass. The first block is the block 20 kilograms. The enlarged block is the block 100 kilograms. It must be mentioned that the kinetic energy depends on a mass [4]. The kinetic energy of the first block is transferred into the ball during the impact. Therefore, the more mass of the block the more kinetic energy is transferred into the ball during the impact. Hence, the ball must get a higher speed because the ball receives more kinetic energy. If the experiment is made in vacuum, then no kinetic energy is lost because of air resistance. The classical mechanics would appear not to contain any explanation what physical processes between the particles of the two impacting bodies may cause the increase of the ball final speed when the mass of the block is increased. The author assumes that the kinetic energy transfer may happen via some quantum entanglement interactions.

One more aspect considered in the experiment is how the ball gains a higher speed. The author assumes that one of two possible processes may happen during the impacts. The first is that the block and the ball get the same acceleration during their mechanical impacts; however, the ball begins to get a higher acceleration after the impacts end.

The second is that the block and the ball get the same acceleration during the impacts; however, the ball continues to move with the same acceleration for a short time after the impacts end. Therefore, the ball is accelerated for a little bit 
longer after the mechanical impact ends when the ball is accelerated by the enlarged block. It should be mentioned that the ball cannot by inertia get a higher speed without some acceleration after the impact between the enlarged block and the ball ends. Based on Newton's first law, all bodies move uniformly (without acceleration) by inertia if no any force is exerted on them.

However, if a distance, which the ball flies over when it is accelerated by the first block, is equal to a distance, which the ball flies over when it is accelerated by the enlarged block, it means that the speeds of the ball before and after the mass increase of the block are equal. It raises a point of eligibility of the formula (4). It is because the impact during the acceleration of the block and the ball is a collision by nature, even though such a collision lasts for a relatively longer time and may not have any shock waves. Another point concerns the kinetic energy transfer from the block to the ball. The enlarged block has more kinetic energy than the first block; therefore, more kinetic energy should be transferred to the ball. The kinetic energy transfer must take place because the block and the ball interact with each other [5]. Notice that the final speeds (speeds after impact) of two bodies, which collide with each other, depend on masses of these bodies and are calculated based on the known formula with the coefficient of restitution in inelastic collisions [6].

The experiment described in this paper is implemented horizontally, but it can be made vertically too.

References:

1. https://www.khanacademy.org/science/physics/forces-newtons-laws/normal-contact-force/a/what-is-normal-force

2. https://en.wikipedia.org/wiki/lmpulse (physics);

3. https://en.wikipedia.org/wiki/Newton's laws of motion;

4. https://en.wikipedia.org/wiki/Kinetic_energy ;

5. https://www.uwsp.edu/cnr-ap/KEEP/nres633/Pages/Unit2/Section-B-Energy-Transfer.aspx

6. https://en.wikipedia.org/wiki/Inelastic collision 